\title{
Cylicocyclus species predominate during shortened egg reappearance period in horses after treatment with ivermectin and moxidectin
}

\author{
D.C.K. van Doorn ${ }^{\mathrm{a}, *}$, H.W. Ploeger ${ }^{\mathrm{a}}$, M. Eysker ${ }^{\mathrm{a}}$, T. Geurden ${ }^{\mathrm{b}}$, J.A. Wagenaar ${ }^{\mathrm{a}}$, \\ F.N.J. Kooyman ${ }^{\mathrm{a}}$ \\ a Department of Infectious Diseases and Immunology, Faculty of Veterinary Medicine, Utrecht University, Yalelaan 1, 3584 CL Utrecht, \\ The Netherlands \\ b Zoetis - Veterinary Medicine Research and Development, Hoge Wei 10, 1930 Zaventem, Belgium
}

\section{A R T I C L E I N F O}

\section{Article history:}

Received 15 November 2013

Received in revised form 3 October 2014

Accepted 4 October 2014

\section{Keywords:}

Cyathostominae

Egg reappearance period

Ivermectin

Moxidectin

Cylicocyclus

Horses

\begin{abstract}
A B S T R A C T
The normal time interval between treatment of horses with a macrocyclic lactone (ML) and reappearance of strongyle eggs in the feces, or 'Egg Reappearance Period (ERP)', is at its shortest 8 weeks for ivermectin (IVM) and 12 weeks for moxidectin (MOX). Nowadays, it is not uncommon to find shorter ERPs, potentially indicating the beginning of the development of drug resistance. Whether all cyathostomin species contribute equally to a shortened ERP is not known. In the present study a Reverse Line Blot (RLB) on individual infective larvae was used to compare species composition before and after ML-treatment in horses with either a normal or a shortened ERP. Species composition was determined for a total of 21 horses on 4 farms and was based on differentiation of approximately 40 larvae per horse per sampling day.

After IVM treatment the shortest ERP was found to be 28-42 days. After MOX treatment the shortest ERP was found to be 42-56 days. The RLB showed that early egg shedding was dominated by species of the genus Cylicocyclus (Cyc.) (Cyc. ashworthi, Cyc. insigne, Cyc. leptostomum and Cyc. nassatus). Before treatment, species composition was generally more diverse with species from several other genera also present. Interestingly, on the farm with the overall shortest ERP and where all horses showed a shortened ERP, more species/genera were present during early egg excretion than on other farms. Results suggest that cyathostomin species within the genus Cylicocyclus contribute to a shortened ERP more than species from other genera. However, if shortening of ERP overall becomes more pronounced and widespread within a farm, species from other genera will also be present during early egg shedding.
\end{abstract}

(c) 2014 Elsevier B.V. All rights reserved.

\footnotetext{
* Corresponding author. Tel.: +31 302532459.

E-mail address: d.c.k.vanDoorn@uu.nl (D.C.K. van Doorn).
}

\section{Introduction}

Cyathostomins are the most prevalent horse helminths with over 50 species described in equids (Lichtenfels et al., 2008). Cyathostomins may cause disease in horses of all ages, but is most often seen as larval cyathostominosis in young horses (Herd, 1986). Worldwide, the control of 
cyathostomin infections depends to a large extent on the use of anthelmintics. However, in many countries drug resistance in equine parasites has become problematic (Drudge et al., 1979; Molento et al., 2008; Rossano et al., 2010). This probably extends to the group of the macrocyclic lactones (MLs) as has been suggested recently by Traversa et al. (2009b), Canever et al. (2013) and Geurden et al. (2014).

Anthelmintic resistance can be diagnosed using the Fecal Egg Count Reduction Test (FECRT) as defined in the WAAVP guidelines (Coles et al., 1992). It is the most widely adopted technique to determine reduced efficacy of an anthelmintic by measuring the reduction in egg counts between day of treatment and 14 days later.

A disadvantage of only determining FECR at 14 days after treatment is that it does not take a shortening of the egg reappearance period (ERP) into account. Initially, an ERP of between 8 and 12 weeks was observed for ivermectin (IVM) (Borgsteede et al., 1993; Parry et al., 1993; Boersema et al., 1996, 1998). For moxidectin (MOX) the ERP was found to be between 12 and 25 weeks when the drug was first introduced (DiPietro et al., 1997; Demeulenaere et al., 1997; Boersema et al., 1998). A shortened ERP may be the first sign of developing resistance (Lyons et al., 2008, 2009) and shortened ERPs are increasingly reported worldwide (DiPietro et al., 1997; von Samson-Himmelstjerna et al., 2007; Lyons et al., 2008, 2010; Molento et al., 2008; Schumacher et al., 2009; Rossano et al., 2010; Geurden et al., 2014).

Because of the large number of cyathostomin species that could be involved in resistance development, it may be interesting to know which species or genera contribute to a shortening of the ERP as different species may have varying pre-patent period (PPP) (Lyons et al., 2011) and as a result of this varying ERP. Unfortunately, it is not possible to monitor by morphological means which of the many species of cyathostomins contribute to a shortened ERP unless horses are necropsied sequentially. However, molecular differentiation of eggs or infective larvae (L3) to species has become feasible (Hodgkinson et al., 2005; Traversa et al., 2007; Cwiklinski et al., 2012), reducing the need for sequential necropsies.

Hodgkinson et al. (2005) used a PCR-ELISA technique for identification of 6 cyathostomin species to study the early strongyle egg shedding after fenbendazole (FBZ) treatment of horses. Within their study egg reappearance per species was described, but no significant changes in species composition before and after treatment were found. Traversa et al. (2009a) found 8 cyathostomin species in pre-treatment pooled egg samples whilst only Coronocyclus (Cor.) labiatus and Cylicostephanus (Cys.) goldi were detected 14 days after treatment with FBZ. Three studies on cyathostomin species following ML treatment were published, two of which used critical tests to determine the effect of MLs on the different cyathostomin species. Cylicocyclus (Cyc.) insigne was the predominant species in 4 yearlings after IVM treatment and only L4 were identified in 4 two-year old horses after MOX treatment (Lyons et al., 2009, 2010). The third study used a Reverse Line Blot (RLB) on pooled egg samples from 4 foals and 4 yearlings (Ionita et al., 2010). They found 11 cyathostomin species before IVM treatment, while 4 weeks after treatment 4 species were found to predominate: Cyc. nassatus, Cys. longibursatus, Cys. calicatus and Cys. minutus.

Studies like those mentioned above are laborious to perform on many horses at the same time. Not surprisingly, data on the temporal reappearance of different cyathostomin species following treatment are still scarce. The aim of the present study was to determine, in 21 horses from 4 different farms, which cyathostomin species contribute to early egg excretion following IVM or MOX treatment.

\section{Materials and methods}

\subsection{Definition of ERP and ERPi}

As there is still no world-wide consensus on the definition of the ERP, we used the following definition. ERP is the time after treatment at which the mean of the individual EPGs return to $10 \%$ or more of the pre-treatment mean fecal egg counts (Borgsteede et al., 1993; von SamsonHimmelstjerna et al., 2007; Larsen et al., 2011). The ERP for individual horses is defined as ERPi.

\subsection{Study 1}

Starting in October 2011 horses (between 4 months and 4 years of age) on 2 farms were investigated for strongyle egg shedding pre- and post-treatment. On farm $1 \mathrm{~A} 11$ horses and on farm 1B 9 horses shedding at least 100 eggs per gram (EPG) were selected and were orally treated (day 0) with $200 \mu \mathrm{g} / \mathrm{kg}$ IVM (Eraquell ${ }^{\circledR}$, VIRBAC, Cacem, Portugal). Fecal samples were collected from all individual horses according to the schedule given in Table 1. On each farm a horse with a regular ERPi and 2 horses with a

Table 1

Mean fecal Egg Count (in eggs per gram) for all farms on all sampling days before and after treatment with either IVM (4 farms) or MOX (2 farms).

\begin{tabular}{|c|c|c|c|c|c|c|c|c|c|c|}
\hline \multirow[t]{2}{*}{ Farms } & \multirow[t]{2}{*}{ Treatment } & \multicolumn{9}{|c|}{ Fecal sampling days } \\
\hline & & PRE & Day 14 & Day 21 & Day 28 & Day 35 & Day 42 & Day 56 & Day 70 & Day 84 \\
\hline $1 \mathrm{~A}$ & IVM & $445 a$ & 0 & 0 & $27 a$ & 136 & $223 a$ & $N$ & $1318 a$ & 641 \\
\hline $1 \mathrm{~B}$ & IVM & $656 a$ & 0 & 0 & 0 & $44 a$ & $100 a$ & $N$ & $517 a$ & 739 \\
\hline $2 \mathrm{~A}$ & IVM & $563 a$ & 0 & 0 & $75 a$ & $154 a$ & $213 a$ & $333 a$ & $N$ & $N$ \\
\hline $2 \mathrm{~A}$ & MOX & $608 a$ & 13 & 0 & $0 \mathrm{a}$ & $38 a$ & $146 a$ & 346 & 788 & $444 a$ \\
\hline $2 B$ & IVM & $458 a$ & 0 & 0 & 0 & 0 & $25 a$ & $50 a$ & $N$ & $N$ \\
\hline $2 B$ & MOX & $650 a$ & 0 & 0 & 0 & 7 & $25 a$ & $125 a$ & $275 a$ & $140 a$ \\
\hline
\end{tabular}

PRE, pre-treatment; $N$, no sampling; a, individual larvae differentiated from selected horses. 
shortened ERPi were chosen for the differentiation of the L3s.

\subsection{Study 2}

Between the end of January and the middle of February 2012 horses (also between 4 months and 4 years of age) on 2 farms (2A and 2B) shedding at least 100 strongyle eggs per gram (EPG) were selected and orally treated (day 0) with either $200 \mu \mathrm{g} / \mathrm{kg}$ IVM (Eqvalan ${ }^{\circledR}$, MERIAL, Toulouse, France) or $400 \mu \mathrm{g} / \mathrm{kg}$ MOX (Equest ${ }^{\circledR}$, ZOETIS, Gerona, Spain). Fecal samples were collected from 6 horses on farm 2A and 6 horses on farm 2B until 56 days after IVM treatment (see Table 1). Also, fecal samples were collected from 6 horses on farm 2A and 7 horses on farm 2B until 84 days after MOX treatment (see Table 1). Farms $2 \mathrm{~A}$ and $2 B$ had been selected from 10 farms that were screened to identify farms with shortened ERP. Farm 2A was selected because all sampled horses showed a shortened ERPi following IVM or MOX treatment, while farm 2B had horses with both regular and shortened ERPi after IVM or MOX treatment.

From farm 2A 6 horses were chosen from which cultured L3s were differentiated, of which 3 had been treated with IVM and 3 with MOX. From farm 2B cultured L3s from 5 horses that were treated with IVM were used for differentiation. Three of these horses had a regular ERPi and 2 a shortened ERPi.

Finally, from the horses treated with MOX on farm 2B, 4 were chosen from which the cultured L3s were differentiated, one horse with a regular and 3 horses with shortened ERPi.

\subsection{Control farm}

In addition to studies 1 and 2 described above, a control farm was included where all sampled horses (between 1 and 4 years of age) showed normal ERPi's. Four horses were chosen from which the L3s were differentiated. The purpose was to compare pre-treatment species composition at farms with shortened ERP with a farm where the ERP was not shortened.

\subsection{Fecal egg counts and culturing larvae}

From all fecal samples egg counts were performed and larval cultures made. Fecal egg counts were performed with a modified McMaster method with saturated $\mathrm{NaCl}$ solution with a detection limit of 50 EPG (study 1 ) or 25 EPG (study 2) (MAFF, 1986).

For larval cultures $25 \mathrm{~g}$ of feces and $2.5 \mathrm{~g}$ of saw-dust was mixed and cultured in glass jars for 7 days at $26^{\circ} \mathrm{C}$. Subsequently, the jars were filled with lukewarm tap water, turned upside down on petri dishes and replenished with water. L3s were harvested the next day and stored in $50 \mathrm{ml}$ clean water at $4^{\circ} \mathrm{C}$ until further use (MAFF, 1986).

\subsection{Samples used for differentiating individual L3s}

Basically, in both studies the sampling days chosen to differentiate L3s were pre-treatment and at the regular
ERP days post-treatment, being 56 days for IVM and 84 days for MOX. If ERPi was shortened, L3s were also differentiated at earlier days post-treatment. Details on specific days when L3s were differentiated are given in Table 1 and in Table S2. Approximately 40 individual L3s per culture were differentiated with the RLB (see below) since only 40 samples can be processed on one mini-blot at a time. This limits the number of individual larvae that can be identified, but still allows a quantitative estimate of the species composition in each fecal sample. Overall, L3s were differentiated to species level from 21 horses at several points in time during the study.

Supplementary Table S2 related to this article can be found, in the online version, at http://dx.doi.org/10.1016/ j.vetpar.2014.10.004.

\subsection{DNA extraction, PCR amplification and Reverse Line Blot}

The RLB was used for the differentiation of individual L3 instead of individual or pooled eggs because they are easier to isolate and the amount of DNA is constant in contrast to eggs in which the amount of DNA depends on the stage of development (Pecson et al., 2006). The RLB is based on hybridization of a species specific probe with an amplified fragment of the Inter Genic Spacer (IGS) region (Traversa et al., 2007). Each individually isolated L3 was lysed in $25 \mu \mathrm{l}$ Worm Lysis Buffer/proteinase $\mathrm{K}$ (van der Veer et al., 2003). The mixture was frozen for $15 \mathrm{~min}$ at $-80^{\circ} \mathrm{C}$ and the L3s were lysed overnight at $56-60^{\circ} \mathrm{C}$. Proteinase $\mathrm{K}$ was heat inactivated the next day at $95^{\circ} \mathrm{C}$ for $15 \mathrm{~min}$. Lysis was done as described by van Doorn et al. (2010), except that L3s were used instead of exsheathed L3s. For a $25 \mu \mathrm{l}$ PCR reaction $2.5 \mu \mathrm{l}$ of lysate was used as template. PCRs on individual larvae were performed with CY26 (5'-GAGCTGGGTTTAGACCGTCGTGAG-3') as a forward and CY18 (5'-CTTAGACATGCATGGCTTAATC-3') as a biotinylated reverse primer in a thermal cycler using the following protocol: $10 \mathrm{~min}$ at $94{ }^{\circ} \mathrm{C}$ and 35 cycles at $94{ }^{\circ} \mathrm{C}$ for $1 \mathrm{~min}, 55^{\circ} \mathrm{C}$ for $1 \mathrm{~min}$, and $72^{\circ} \mathrm{C}$ for $2 \mathrm{~min}$, followed by a final extension at $72{ }^{\circ} \mathrm{C}$ for $7 \mathrm{~min}$. For RLB $10 \mu \mathrm{l}$ of the IGS amplicon per lane was used for hybridization with 21 species specific probes and 1 cyathostomin catch-all as described by Cwiklinski et al. (2012). The 21 species specific probes included Coronocyclus (Cor) coronatus, Cor. labiatus, Cor. labratus, Cyathostomum (Cya.) catinatum, Cya. pateratum, Cya. tetracanthum, Cylicocyclus (Cyc.) ashworthi, Cyc. auriculatus, Cyc. bidentatus, Cyc. insigne, Cyc. leptostomum, Cyc. nassatus, Cyc. radiatus, Cylicodontophorus bicoronatus, Cylicostephanus (Cys.) calicatus, Cys. goldi, Cys. longibursatus, Cys. minutus, Parapoteriostomum mettami, Poteriostomum imparidentatum and Tridentoinfundibulum gobi. If the PCR product of an individual L3 hybridized with the catch-all probe, but with none of the 21 specific probes, the L3 was identified as "other cyathostomin". Hybridized products were detected with streptavidin-POD conjugate, followed by incubation in ECL detection reagent and exposure to Amersham Hyperfilm ECL. 


\section{Results}

\subsection{FEC and ERP within study 1 and study 2}

The FEC means on all sample days of the 4 farms are given in Table 1. All individual FEC data are given in the supplementary data (Table S1). Table 1 shows that the shortest ERP, based on mean FEC and following IVM treatment was 28 days and following MOX treatment 42 days, both on farm $2 \mathrm{~A}$.

Supplementary Table S1 related to this article can be found, in the online version, at http://dx.doi.org/10.1016/ j.vetpar.2014.10.004.

\subsection{Species composition at farms $1 A$ and $1 B$ (study 1)}

In the pre-treatment samples we found 11 species (mainly Cya. catinatum, Cya. pateratum, Cys. goldi, Cyc. ashworthi, Cyc. leptostomum and Cyc. nassatus) and 9 species (mainly Cys. longibursatus, Cyc. ashworthi, Cyc. leptostomum, and Cyc. nassatus) at farm $1 \mathrm{~A}$ and $1 \mathrm{~B}$, respectively. On farm $1 \mathrm{~A}$ the ERP was 35 days (Table 1 ), the ERPi varied between 28 and 35 days for individual horses (Table S1). On farm $1 \mathrm{~B}$ the ERP was 42 days and the ERPi varied between 35 and 42 days. When the species from the same genus were grouped together it was shown that Cylicocyclus spp. contributed most to earlier egg shedding after treatment (Fig. 1 and Table S2). On farm 1A the first eggs found after treatment were from Cyc. ashworthi and Cyc. leptostomum. At farm 1B the first eggs after treatment were from Cyc. insigne, Cyc. nassatus together with Cys. longibursatus and $P$. mettami. Subsequently, the species composition became more diverse, with additionally Cya. catinatum, Cya. pateratum and "other species" (not included in the panel of 21 species). For reasons unknown, some cultured larvae did not result in useful PCR products. On one sample day from a pre-treatment culture from farm $1 \mathrm{~B}$, we did not gain differentiation results (Table S2).

\subsection{Species composition at farms $2 A$ and $2 B$ (study 2)}

In the pre-treatment samples of study 2 we identified 9 species (mainly Cys. longibursatus, Cyc. ashworthi, Cyc. leptostomum and Cyc. nassatus) on farm $2 \mathrm{~A}$ and 10 species on farm 2B (mainly Cys. longibursatus, Cyc. ashworthi, Cyc. leptostomum and Cyc. nassatus). On farm 2A, the ERP after IVM treatment was 28 days and after MOX treatment 42 days (Table 1). On farm 2B, the ERP after both IVM and MOX treatment was 56 days (Table 1 ).

As in study 1 the genus Cylicocyclus contributed most to shortened ERP after treatment with either IVM or MOX (Figs. 2 and 3 and Table S2). On farm 2A Cyc. ashworthi, Cyc. leptostomum, Cyc. nassatus and Cys. longibursatus were the first species to return to egg shedding after IVM treatment. On both farms 2A and 2B Cylicocyclus species predominated until day 56 after IVM treatment (Fig. 2). However, the cultures from farm 2A on days 42 and 56 also contained L3 from the genera Cyathostomum and Cylicostephanus, while on farm 2B the cultures consisted exclusively of Cylicocyclus species. Following MOX treatment on farm 2A mainly Cyc. nassatus and one "other" L3 were found at day 28 . The L3
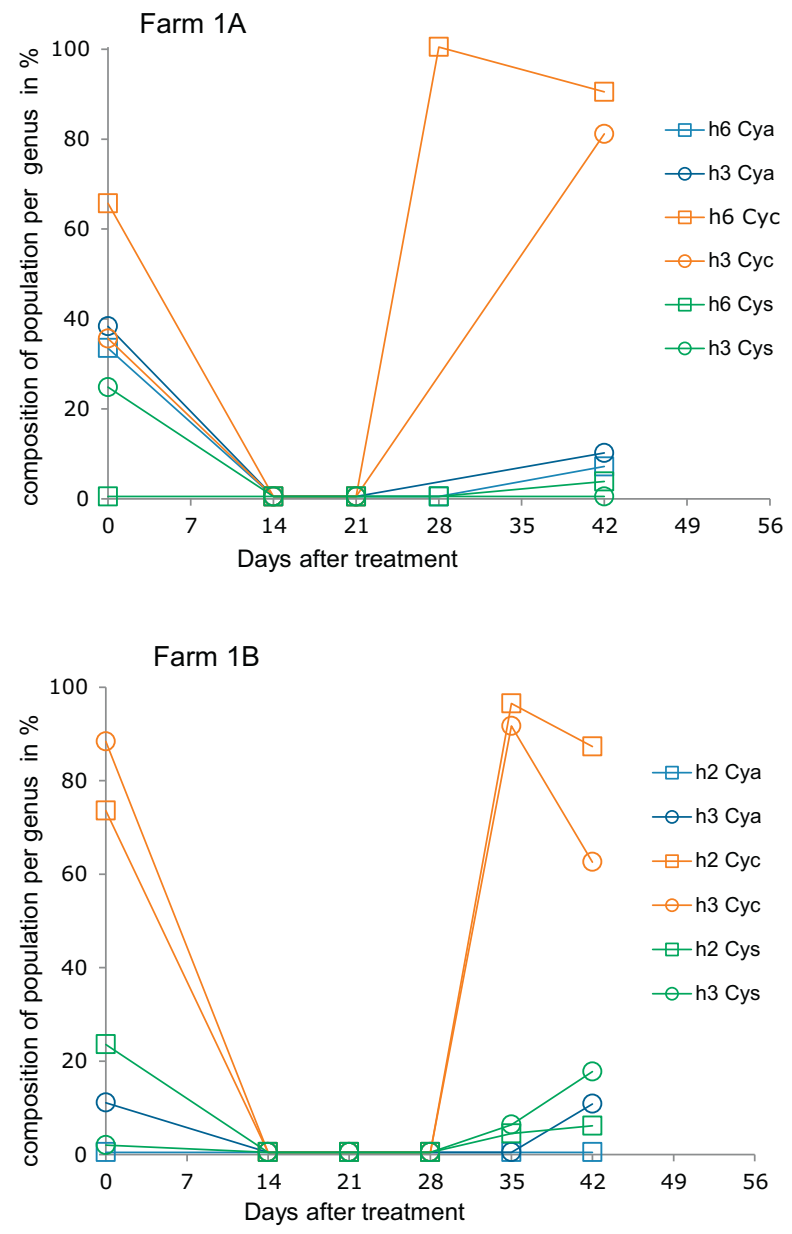

Fig. 1. Genus composition of the L3s obtained before and after IVM treatment from individual horses with a shortened egg reappearance period (ERPi) at farm $1 \mathrm{~A}$ ( 2 horses) and $1 \mathrm{~B}$ ( 2 horses). h\#, horse ID number. When no $L 3 s$ were present $(L P G=0)$, species composition was set at 0 for all genera. The cyathostomin species from the same genus were combined, the 3 most prominent genera are given. Cya, Cyathostomum (blue); Суc, Cylicocyclus (orange); Cys, Cylicostephanus (green). (For interpretation of the references to color in figure legend, the reader is referred to the web version of the article.)

from the MOX treated horses at day 42 on both farms were mainly Cylicocyclus spp. which remained so for the entire period until day 84 (Fig. 3). Additionally, at farm 2A L3 of the genera Coronocyclus and Cylicostephanus were found on day 42 after MOX treatment.

\subsection{Species composition at the control farm}

In the pre-treatment samples from the control farm 9 species were identified, which were mainly Cya. catinatum, Cyc. leptostomum and Cyc. nassatus (see Table S2). Overall, the same species composition was found as was found pre-treatment on the farms from studies 1 and 2 .

\section{Discussion}

The main results in the present study were that the mean ERP after IVM was between 28 and 42 days on the 

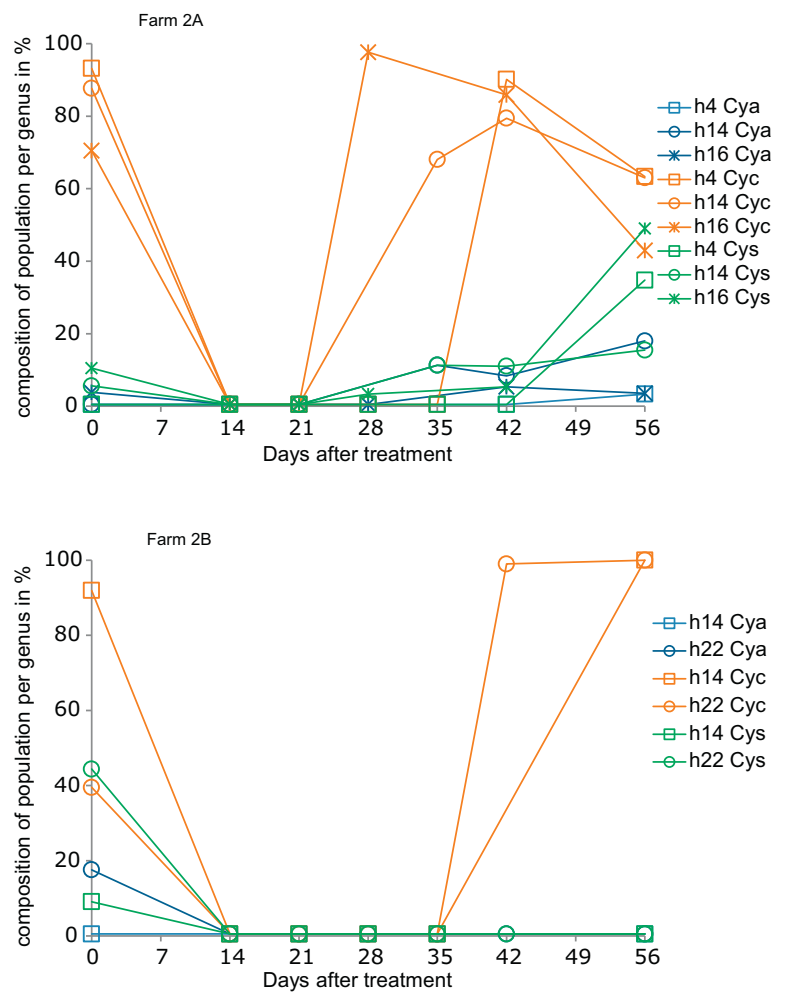

Fig. 2. Genus composition of the L3s obtained before and after IVM treatment in horses with a shortened egg reappearance period (ERPi) at farm $2 \mathrm{~A}(n=3)$ and $2 \mathrm{~B}(n=2)$. h\#, horse ID number. When no L3s were present $(\mathrm{LPG}=0)$, species composition was set at 0 for all genera. The cyathostomin species from the same genus were combined, the 3 most prominent genera are given. Cya, Cyathostomum (blue); Cyc, Cylicocyclus (orange); Cys, Cylicostephanus (green). (For interpretation of the references to color in figure legend, the reader is referred to the web version of the article.)

4 farms examined and after MOX between 42 and 56 days on the 2 examined farms. The first eggs reappearing after treatment with either IVM or MOX were identified to be from species within the genus Cylicocyclus.

On farm 2A all sampled horses showed a shortened egg reappearance, while on farm $2 B$ several horses still had a normal ERPi. The mean ERP was for farm $2 \mathrm{~A}$ also shorter than for farm 2B. This might reflect lesser efficacy of MLs on farm 2A compared with farm 2B. This is supported by the fact that on farm $2 \mathrm{~A}$ more genera were present in larval cultures early after treatment than on farm 2B. Compared to results after IVM treatment, results after MOX treatment were somewhat more variable, but still Cylicocyclus species were predominating in horses with a shortened ERPi. Interestingly, sometimes Cylicocyclus species that were abundant before treatment seemed to be replaced by other Cylicocyclus species after treatment. Our findings are generally in line with Lyons et al. (2009, 2010). They used critical tests and observed that Cylicocyclus species predominate in horses following IVM or MOX treatment. Ionita et al. (2010) differentiated cyathostomin species before and after treatment in pools with different numbers of eggs, making a comparison difficult. Further
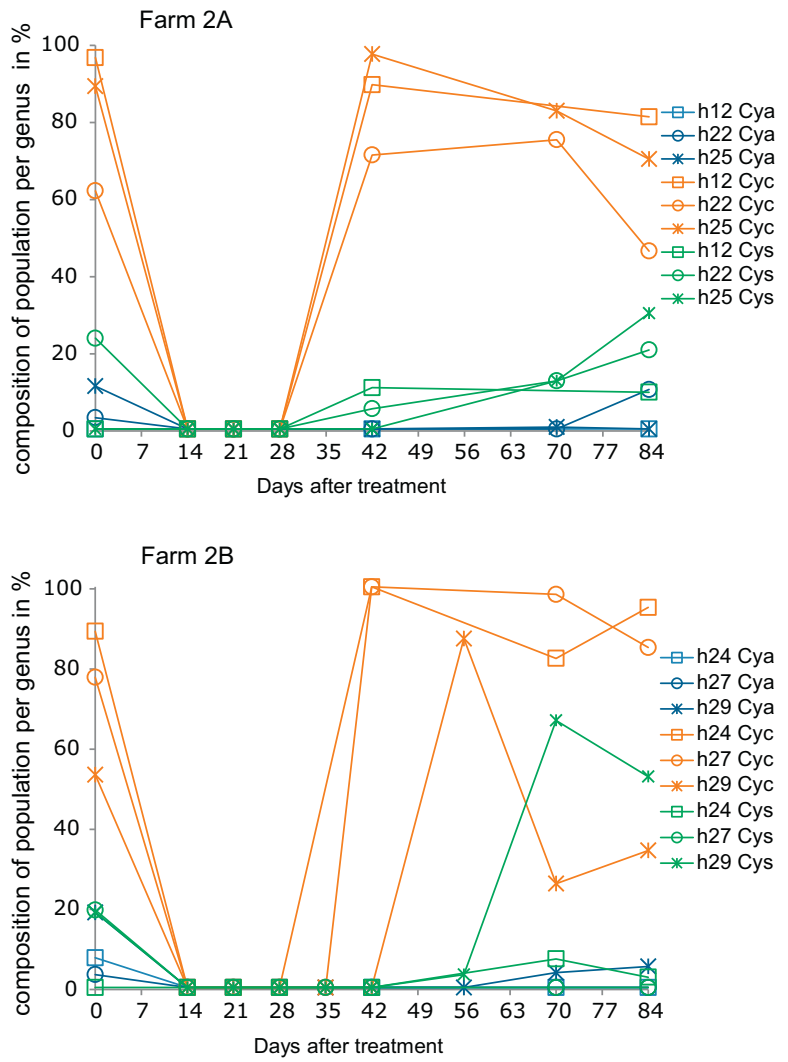

Fig. 3. Genus composition of the L3s obtained before and after MOX treatment in horses with a shortened egg reappearance period (ERPi) at farm $2 \mathrm{~A}(n=3)$ and $2 \mathrm{~B}(n=3)$. h\#, horse ID number. When no L3s were present ( $L P G=0)$, species composition was set at 0 for all genera. The cyathostomin species from the same genus were combined, the 3 most prominent genera are given. Cya, Cyathostomum (blue); Сyc, Cylicocyclus (orange); Cys, Cylicostephanus (green). (For interpretation of the references to color in figure legend, the reader is referred to the web version of the article.)

studies on more farms and in other countries will show if the genus Cylicocyclus is always involved in shortened ERP.

How can we explain the rapid reappearance of eggs in horses within this study? At least on farms $2 \mathrm{~A}$ and $2 \mathrm{~B}$ egg shedding after treatment must have originated from adults that survived treatment as immatures, because horses were treated in January and February and were stabled from November until April and during housing in winter acquisition of infection is negligible.

A 1st explanation could be a structural shift in the cyathostomin species composition after prolonged ML usage. In the present study species composition before treatment did not differ much between farms with varying degrees of shortened ERPs. This was also the case within farms between horses with and without a shortened ERPi. Moreover, on the control farm, with all horses having a regular ERPi, we found the same cyathostomin species composition as on the other farms. These observations fit those from Chapman et al. (2002), who found that after 20 years of treatments with MLs the diversity of cyathostomin species remained the same, without major shifts in the prevalence and ranking of species. 
A 2nd explanation could be reduced susceptibility of L4s for MLs. After IVM (Lyons et al., 2009) or MOX treatment (Lyons et al., 2010) luminal L4s were found 6 days after treatment. This may suggest that beginning resistance is first observed in L4s. A reason for resistance in L4s might be that L3s and L4s are partly protected by their mucosal location and/or their immobility. This is in contrast with the luminal L4s and the adults. Another explanation might be that luminal L4s have a different metabolism and genetic expression profiles which make them more liable to develop resistance. However, we can only speculate why adults may be less sensitive to develop resistance compared with L4s.

A 3rd explanation for rapid reappearance after ML treatment might be selection for a shorter parasitic phase of the lifecycle. A shorter parasitic phase may result in less exposure to ML treatments and consequently increased survival chances. It has been observed that Cylicocyclus spp. develop slowly in the submucosa, in eight weeks or more, compared to other cyathostomin species with faster development like Cys. longibursatus, Cya. catinatum, Cor. coronatum, Cys. minutus and Cys. calicatus (Tiunov, 1951 referenced by Ogbourne, 1975, 1978). Yet, Cylicocyclus spp. predominated in horses with shortened ERP. So, if this explanation is true, it remains to be elucidated why Cylicocyclus species might be more easily selected for a shorter parasitic phase than species from other cyathostomin genera.

For now it remains difficult to explain why mainly species within the genus Cylicocyclus were found early after ML treatment. Cylicocyclus species migrate deeper into the submucosa of the large intestine and they remain there for a longer part of their development compared to other species (Tiunov, 1951 referenced by Ogbourne, 1975, 1978). If this means that the period of the vulnerable luminal L4 is correspondingly shorter, this might play a role in the early reappearance of this genus.

Finally, the RLB proved to be a very useful technique to sequentially monitor species composition following $\mathrm{ML}$ treatment. Probes for 21 species were used whereas over 50 species of cyathostomins have been described. In 53\% of the differentiated larval batches no "other" species were found and when a batch did contain "other" species this constituted between 3 and $24 \%$ of the total number of larvae. So, species specific probes for the 21 most common cyathostomin species apparently sufficed to determine the overall species composition although relatively rare species may remain undetected. On the other hand, the RLB technique for use on individual larvae is not a high throughput method. Therefore, we could not apply this technique on all larval cultures from all horses involved in this study. Nonetheless, using the RLB on individual larvae can provide a wealth of sequential data on species compositions without having to resort to necropsies.

\section{Conclusion}

Within 4-8 weeks after ML treatment Cylicocyclus species were present far more often than species from other genera. On the farm with the most pronounced ERP shortening $(2 A)$, species from other genera were also found early after treatment.

\section{Conflict of interest statement}

This study was co-financed by Zoetis. The interpretation of the results was not influenced by Zoetis. Project code Zoetis 70AQ055200 or study number Zoetis 5253R-50-11-200.

\section{Acknowledgements}

We would like to thank the farm managers for their cooperation. For technical assistance we thank S. Rodenberg and B. van $t$ Klooster. We also thank C. Klaassen and C. Felix, students from HAS Dronten, and Dr. E.G. Dingboom and the students from HAS Den Bosch.

\section{References}

Boersema, J.H., Eysker, M., Maas, J., van der Aar, W.M., 1996. Comparison of the reappearance of strongyle eggs on foals, yearlings and adult horses after treatment with ivermectin or pyrantel. Vet. Q. 18, 7-9.

Boersema, J.H., Eysker, M., van der Aar, W.M., 1998. The reappearance of strongyle eggs in the faeces of horses after treatment with moxidectin. Vet. Q. 20, 15-17.

Borgsteede, F.H.M., Boersma, J.H., Gaasenbeek, C.P., van der Burg, W.P., 1993. The reappearance of eggs in faeces of horses after treatment with ivermectin. Vet. Q. 15, 24-26.

Canever, R.J., Braga, P.R.C., Boeckh, A., Grycajuck, M., Bier, D., Molento, M.B., 2013. Lack of Cyathostomin sp. reduction after anthelmintic treatment in horses in Brazil. Vet. Parasitol. 194, 35-39.

Chapman, M.R., French, D.D., Klei, T.R., 2002. Gastrointestinal helminths of ponies in Louisiana: a comparison of species currently prevalent with those present 20 years ago. J. Parasitol. 6, 1130-1134.

Coles, G.C., Bauer, C., Borgsteede, F.H., Geerts, S., Klei, T.R., Taylor, M.A., Waller, P.J., 1992. World Association for the Advancement of Veterinary Parasitology (W.A.A.V.P.) methods for the detection of anthelmintic resistance in nematodes of veterinary importance. Vet. Parasitol. 44, 35-44

Cwiklinski, K., Kooyman, F.N.J., van Doorn, D.C.K., Matthews, J.B., Hodgkinson, J.E., 2012. New insights into sequence variation in the IGS region of 21 cyathostomin species and the implication for molecular identification. Parasitology 139, 1063-1073.

Demeulenaere, D., Vercruysse, J., Dorny, P., Claerebout, E., 1997. Comparative studies of ivermectin and moxidectin in the control of naturally acquired cyathostome infections in horses. Vet. Rec. 15, 383-386.

DiPietro, J.A., Hutchens, D.E., Lock, T.F., Walker, K., Paul, A.J., Shipley, C., Rulli, D., 1997. Clinical trial of moxidectin oral gel in horses. Vet. Parasitol. 72, 167-177.

Drudge, J.H., Lyons, E.T., Tolliver, S.C., 1979. Benzimidazole resistance of equine strongyles-critical tests of six compounds against population B. Am. J. Vet. Res. 40, 590-594.

Geurden, T., Doorn, D.C.K., Claerebout, E., Kooyman, F.N.J., De Keersmaecker, S., Vercruysse, J., Besognet, B., Vanimisetti, B., Frangipane di Regalbono, A., Beraldo, P., Di Cesare, A., Traversa, D., 2014. Decreased strongyle egg re-appearance period after treatment with ivermectin and moxidectin in horses in Belgium, Italy and The Netherlands. Vet. Parasitol., http://dx.doi.org/10.1016/j.vetpar.2014.04.013 (in press).

Herd, R.P., 1986. Epidemiology and control of equine strongylosis at Newmarket. Equine Vet. J. 18, 447-452.

Hodgkinson, J.E., Freeman, K.L., Lichtenfels, J.R., Palfreman, S., Love, S., Matthews, J.B., 2005. Identification of strongyle eggs from anthelmintic-treated horses using a PCR-ELISA based on intergenic DNA sequences. Parasitol. Res. 95, 287-292.

Ionita, M., Howe, D.K., Lyons, E.T., Tolliver, S.C., Kaplan, R.M., Mitrea, I.L., Yeargan, M., 2010. Use of a reverse line blot assay to survey small strongyle (Strongylida: Cyathostominae) populations in horses before and after treatment with ivermectin. Vet. Parasitol. 168, 332-337.

Larsen, M.L., Ritz, C., Petersen, S.L., Nielsen, M., 2011. Determination of ivermectin efficacy against cyathostomins and Parascaris equorumon horse farms using selective therapy. Vet. J. 188, 44-47.

Lichtenfels, J.R., Kharchenko, V.A., Dvojnos, G.M., 2008. Illustrated identification keys to strongylid parasites of horses, zebras and asses. Vet. Parasitol. 156, 4-161. 
Lyons, E.T., Tolliver, S.C., Ionita, M., Lewellen, A., Collins, S.S., 2008. Field studies indicating reduced activity of ivermectin on small strongyles in horses on a farm in Central Kentucky. Parasitol. Res. 103, 209-215.

Lyons, E.T., Tolliver, S.C., Collins, S.S., 2009. Probable reason why small strongyle EPG counts are returning 'early' after ivermectin treatment of horses on a farm in Central Kentucky. Parasitol. Res. 104, 569-574.

Lyons, E.T., Tolliver, S.C., Kuzmina, T.A., Collins, S.S., 2010. Critical tests evaluating efficacy of moxidectin against small strongyles in horses from a herd for which reduced activity had been found in field tests in Central Kentucky. Parasitol. Res. 107, 1495-1498.

Lyons, E.T., Kuzmina, T.A., Tolliver, S.C., Collins, S.S., 2011. Observations on development of natural infection and species composition of small strongyles in young equids in Kentucky. Parasitol. Res. 109, 1529-1535

Ministry of Agriculture Fisheries and Food (MAFF), 1986. Manual of Veterinary Parasitological Laboratory Techniques: Reference Book 418 , 3rd ed. HMSO Books, London.

Molento, M.B., Antunes, J., Bentes, R.N., Coles, G.C., 2008. Anthelmintic resistant nematodes in Brazilian horses. Vet. Rec. 162, 384-385.

Ogbourne, C.P., 1975. Epidemiological studies on horses infected with nematodes of the family Trichonematidae (Witenberg, 1925). Int. J. Parasitol. 5, 667-672.

Ogbourne, C.P., 1978. Pathogenesis of Cyathostome (Trichonema) Infections of the Horse. A review. CIH Miscellaneous Publication No. 5

Parry, J.M., Fisher, M.A., Grimshaw, W.T.R., Jacobs, D.E., 1993. Anthelmintic dosing intervals for horses: comparison of three chemical groups. Vet. Rec. 133, 346-347.

Pecson, B.M., Barrios, J.A., Johnson, D.R., Nelson, K.L., 2006. A real time PCR method for quantifying viable Ascaris eggs using the first internally transcribed spacer region of ribosomal DNA. Appl. Environ. Microb. 72, 7864-7872.

Rossano, M.G., Smith, A.R., Lyons, E.T., 2010. Shortened strongyle-type-egg reappearance periods in naturally infected horses treated with moxidectin and failure of a larvicidal dose of fenbendazole to reduce fecal egg counts. Vet. Parasitol. 173, 349-352.

Schumacher, J., Livesey, L., DeGraves, F., Blagburn, B., Ziska, M., Caldwell, M., Brock, K., 2009. Efficacy of moxidectin against cyathostomins after long-term use in a large herd of draught horses with a high stocking density. Vet. Rec. 164, 652-654.

Traversa, D., Iorio, R., Klei, T.R., Kharchenko, V.A., Gawor, J., Otranto, D., Sparagano, O.A.E., 2007. New method for simultaneous species-specific identification of equine strongyles (Nematoda, Strongylida) by reverse line blot hybridization. J. Clin. Microbiol. 45, 2937-2942.

Traversa, D., Iorio, R., Otranto, D., Giangaspero, A., Milillo, P., Klei, T.R., 2009a. Species-specific identification of equine cyathostomes resistant to fenbendazole and susceptible to oxibendazole and moxidectin by macroarray probing. Exp. Parasitol. 121, 92-95.

Traversa, D., von Samson-Himmelstjerna, G., Demeler, J., Milillo, P., Schürmann, S., Barnes, H., Otranto, D., Perrucci, S., di Regalbono, A.F. Beraldo, P., Boeckh, A., Cobb, R., 2009b. Anthelmintic resistance in cyathostomin populations from horse yards in Italy, United Kingdom and Germany. Parasites Vectors 2 (September (Suppl. 2)), S2.

van der Veer, M. Kanobana, K. Ploeger, H.W., de Vries, E., 2003. Cytochrome oxidase $C$ subunit 1 polymorphisms show significant differences in distribution between a laboratory maintained population and a field isolate of Cooperia oncophora. Vet. Parasitol. 116, 231-238.

van Doorn, D.C.K., Kooyman, F.N.J., Eysker, M., Hodgkinson, J.E., Wagenaar, J.A., Ploeger, H.W., 2010. In vitro selection and differentiation of ivermectin resistant cyathostomin larvae. Vet. Parasitol. 174, 292-299.

von Samson-Himmelstjerna, G., Fritzen, B., Demeler, J., Schurmann, S. Rohn, K., Schnieder, T., Epe, C., 2007. Cases of reduced cyathostomin egg-reappearance period and failure of Parascaris equorum egg count reduction following ivermectin treatment as well as survey on pyrantel efficacy on German horse farms. Vet. Parasitol. 144, 74-80. 